\title{
Exact Solution of a Three-Dimensional Dimer System
}

\author{
H. Y. Huang, ${ }^{1}$ V. Popkov, ${ }^{2,3}$ and F. Y. Wu ${ }^{1}$ \\ ${ }^{1}$ Department of Physics and Center for Interdisciplinary Research in Complex Systems, \\ Northeastern University, Boston, Massachusetts 02115 \\ ${ }^{2}$ Center for Theoretical Physics, Seoul National University, Seoul 151-742, Korea \\ ${ }^{3}$ Institute for Low Temperature Physics, Kharkov, Ukraine (permanent address)
}

\begin{abstract}
We consider a three-dimensional lattice model consisting of layers of vertex models coupled with interlayer interactions. For a particular non-trivial interlayer interaction between charge-conserving vertex models and using a transfer matrix approach, we show that the eigenvalues and eigenvectors of the transfer matrix are related to those of the two-dimensional vertex model. The result is applied to analyze the phase transitions in a realistic threedimensional dimer system.
\end{abstract}

$05.50 .+\mathrm{q}$ 
An outstanding unsolved problem in the statistical mechanics of critical phenomena is the pursuit of exact solutions for realistic three-dimensional $(3 D)$ systems. While a large number of $2 D$ systems have yielded to analyses [1], only a limited number of $3 D$ systems have been solved. They include the $3 D$ Ising model solved by Suzuki [2], the Zamolodchikov model [3] solved by Baxter [4] and its more recent $N$-state extension by Bazhanov and Baxter [5]. However, these models invariably suffer defects in one way or another: The Suzuki model turns out to be a $2 D$ system in disguise, while the Zamolodchikov model and its extension involve unphysical negative Boltzmann weights. Similarly, a continuous string model in general $d$ dimensions solved by two of us [6] also involves negative weights. The solution of realistic physical $3 D$ models has remained very much an open problem.

One approach toward solving realistic $3 D$ models is to build from $2 D$ systems. Indeed, such an approach has been suggested [7] and applied with some success recently [8.9]. However, in these considerations much attention has been placed to the algebraic structure of the transfer matrix and the associated Yang-Baxter equation, to the extent of masking the elegance of the solution. Here, we present a more general formulation, and deduce its solution via an alternate and yet much simpler consideration. The result is applied to analyze a realistic layered $3 D$ dimer system.

Consider a simple-cubic lattice $\mathcal{L}$ of size $K \times M \times N$ with periodic boundary conditions. Regard $\mathcal{L}$ as consisting of $K$ copies of square lattices, of $M$ rows and $N$ columns each and stacked together as shown in Fig. 1. For simplicity, we shall speak of the square lattices as "layers" of $\mathcal{L}$. Label sites of $\mathcal{L}$ by indices $\{m, j, k\}$, with $1 \leq m \leq M, 1 \leq j \leq N$ and $1 \leq k \leq K$. Within each layer of $\mathcal{L}$ define a $2 D q$-state vertex model whose lattice edges can be in $q$ distinct states. Label the state of the horizonal (vertical) edge incident at the site $\{m, j, k\}$ in the direction of, say, decreasing $\{m, j\}$ by $\alpha_{m j k}\left(\beta_{m j k}\right)$. It is convenient at times to suppress the subscripts $m$ and/or $k$ by adopting the notation

$$
\beta_{m+1, j, k} \rightarrow \beta_{j}^{\prime}, \quad \beta_{m, j, k+1} \rightarrow \tilde{\beta}_{j},
$$

and similarly for the $\alpha$ 's. Associate vertex weight $W_{m j k}$ to site $\{m, j, k\}$ which is a function 
of the configuration $C_{m, j, k} \equiv\left\{\alpha_{m j k}, \beta_{m j k}, \alpha_{m, j+1, k}, \beta_{m+1, j, k}\right\} \rightarrow\left\{\alpha_{j}, \beta_{j}, \alpha_{j+1}, \beta_{j}^{\prime}\right\}$ of the four edges incident at the site $\{m, j, k\}$. Let the $\{m, j\}$ sites of two adjacent layers $k$ and $k+1$ interact with a Boltzmann factor $B_{m j k}$ which in the most general case is a function of the configurations $C_{m, j, k}$ and $C_{m, j, k+1}$ of the eight edges incident to the two sites. Then, the problem at hand is the evaluation of the partition function

$$
Z_{M N K}=\sum_{\alpha_{m j k}} \sum_{\beta_{m j k}} \prod_{k=1}^{K} \prod_{m=1}^{M} \prod_{j=1}^{N}\left(B_{m j k} W_{m j k}\right)
$$

where the summations are taken over all edge states $\alpha_{m j k}$ and $\beta_{m j k}$, and the per-site "free energy" for any $K$

$$
f_{K}=K^{-1} \lim _{M, N \rightarrow \infty}(M N)^{-1} \ln Z_{M N K}
$$

The transfer matrix: The partition function (2) can be evaluated by applying a transfer matrix in the vertical direction. In a horizontal cross section of $\mathcal{L}$ there are $N K$ vertical edges. Let $\left\{\beta_{m}\right\}=\left\{\beta_{m j k} \mid 1 \leq j \leq N, 1 \leq k \leq K\right\}, 1 \leq m \leq M$ denote the states of these $N K$ vertical edges, and define a $2^{N K} \times 2^{N K}$ matrix $\mathbf{T}$ with elements

$$
T\left(\left\{\beta_{m}\right\},\left\{\beta_{m+1}\right\}\right)=\sum_{\alpha_{m j k}} \prod_{k=1}^{K} \prod_{j=1}^{N}\left(B_{m j k} W_{m j k}\right), \quad 1 \leq m \leq M .
$$

Then one has

$$
\begin{aligned}
Z_{M N K} & =\sum_{\beta_{m j k}} \prod_{m=1}^{M} T\left(\left\{\beta_{m}\right\},\left\{\beta_{m+1}\right\}\right) \\
& =\operatorname{Tr} \mathbf{T}^{M} \\
& \sim \Lambda_{\max }^{M}
\end{aligned}
$$

where $\Lambda_{\max }$ is the largest eigenvalue of $\mathbf{T}$.

It is clear that we need to restrict considerations to models which are soluble when the interlayer interaction is absent, or $B_{m j k}=1$. This leads us to build $3 D$ systems from soluble $2 D$ models. It is also clear that the interlayer interaction $B_{m j k}$ should be such that the overall interlayer factor $\prod_{m, j, k} B_{m j k}$ can be conveniently treated. For this purpose we restrict considerations to $2 D$ charge-conserving models. 
For definiteness let the labels $\alpha_{m j k}$ and $\beta_{m j k}$ take on a set $I$ of $q$ integral values. For example, one can take $I=\{+1,-1\}$ for $q=2$ and $I=\{+1,0,-1\}$ for $q=3$. A $2 D$ vertex model is charge-conserving if its vertex weights are non-vanishing only when

$$
\alpha_{j}+\beta_{j}=\alpha_{j+1}+\beta_{j}^{\prime} \quad(\text { charge conservation })
$$

holds at all sites. Examples of charge conserving models are the $q=2$ ice-rule models [10], the $q$-state string model [11], the $q=3$ Izergin-Korepin model [12], and others [13].

A direct consequence of the charge-conserving rule (6) is deduced by summing (6) from $j=1$ to $j=N$. This yields

$$
y_{k}=\frac{1}{N} \sum_{j=1}^{N} \beta_{j}=\frac{1}{N} \sum_{j=1}^{N} \beta_{j}^{\prime}
$$

showing that the quantity $-1 \leq y_{k} \leq 1$ is independent of $m$. (For ice-rule models this fact is well-known.)

Next one introduces the interlayer interaction [7] 9]

$$
B_{m j k}=\exp \left(h\left(\alpha_{j} \tilde{\beta}_{j}-\tilde{\alpha}_{j+1} \beta_{j}^{\prime}\right)\right) .
$$

Since the negation of $h$ corresponds to a reversal of the layer numberings, without loss of generality we can take $h \geq 0$. We now show quite generally that the interlayer interaction (8) leads to a considerable simplification of the transfer matrix. Consider first the product

$$
\prod_{j=1}^{N} B_{m j k}=\exp \left(h \sum_{j=1}^{N}\left(\alpha_{j} \tilde{\beta}_{j}-\tilde{\alpha}_{j+1} \beta_{j}^{\prime}\right)\right) .
$$

Summing over (6), or $\alpha_{i}+\beta_{i}=\alpha_{i+1}+\beta_{i}^{\prime}$, for $i=\{1, j-1\}$ and $i=\{j+1, N\}$ for the layer $k+1$, one obtains, respectively, the identities

$$
\begin{gathered}
\alpha_{j}=\alpha_{1}+\sum_{i=1}^{j-1}\left(\beta_{i}-\beta_{i}^{\prime}\right), \quad j=2,3 \cdots, N \\
\tilde{\alpha}_{j+1}=\tilde{\alpha}_{1}-\sum_{i=j+1}^{N}\left(\tilde{\beta}_{i}-\tilde{\beta}_{i}^{\prime}\right), \quad j=1,2, \cdots, N-1
\end{gathered}
$$

where we have used $\tilde{\alpha}_{N+1}=\tilde{\alpha}_{1}$. Substituting (10) into (9) and making use of the identity $\sum_{j=2}^{N} \sum_{i=1}^{j-1}=\sum_{i=1}^{N-1} \sum_{j=i+1}^{N}$ in the first summation in (9), one arrives after a little algebra at 


$$
\prod_{j=1}^{N} B_{m j k}=\exp \left(N h\left(\alpha_{1} \tilde{y}-\tilde{\alpha}_{1} y\right)+N h\left[f(\beta, \tilde{\beta})-f\left(\beta^{\prime}, \tilde{\beta}^{\prime}\right)\right]\right),
$$

where $f(\beta, \tilde{\beta}) \equiv \sum_{j=2}^{N} \sum_{i=1}^{j-1} \beta_{i} \tilde{\beta}_{j}$. The numerical factor $f(\beta, \tilde{\beta})$, which is defined for each fixed $m$, is cancelled in the further product

$$
\prod_{m=1}^{M} \prod_{j=1}^{N} B_{m j k}=\prod_{m=1}^{M} \exp \left(N h\left(\alpha_{m, 1, k} y_{k+1}-\alpha_{m, 1, k+1} y_{k}\right)\right) .
$$

As a result, only the conserved quantities $y_{k}$ and the state $\alpha_{m, 1, k}$ of the extremites of a row of horizontal edges appear in the product $(\sqrt{12})$. This leads us to rewrite the partition function (2) as

$$
Z_{M N K}=\operatorname{Tr}\left(\mathbf{T}^{\text {eff }}\right)^{M}
$$

where $\mathbf{T}^{\text {eff }}$ is a matrix with elements

$$
T^{\mathrm{eff}}\left(\left\{\beta_{m}\right\},\left\{\beta_{m+1}\right\}\right)=\sum_{\alpha_{m j k}} \prod_{k=1}^{K}\left(e^{N h \alpha_{m, 1, k}\left(y_{k+1}-y_{k-1}\right)} \prod_{j=1}^{N} W_{m j k}\right) .
$$

The problem is now reduced to one of finding the largest eigenvalue of $\mathbf{T}^{\mathrm{eff}}$. In fact, expression (11) shows that $\mathbf{T}^{\mathrm{eff}}$ is related to $\mathbf{T}$ by a similarity transformation $\mathbf{T}^{\mathrm{eff}}=\mathbf{S} \mathbf{T} \mathbf{S}^{-1}$ where $\mathbf{S}$ is diagonal. It follows that $\mathbf{T}$ and $\mathbf{T}^{\mathrm{eff}}$ have the same eigenvalues, and their eigenvectors are related. The task is now considerably simpler since one needs only to keep track of the $2 D$ system. The problem is solved if the eigenvalues of the transfer matrix for the $2 D$ vertex model can be evaluated for fixed $y_{k}$ and $\alpha_{m, 1, k}$.

The ice-rule model: To illustrate the usefulness of this formulation, we now apply it to layers of ice-rule model with vertex weights $\left\{\omega_{1}, \omega_{2}, \ldots, \omega_{6}\right\}$ (for standard notations relevant to present discussions see, for example, [10]). Let $\alpha=+1(-1)$ denote arrows pointing toward right (left), and $\beta=+1(-1)$ arrows pointing up (down). Then one verifies that the charge-conserving condition (ब) is satisfied with $y_{k}=1-2 n_{k} / N$, where $n_{k}$ is the number of down arrows in a row of vertical edges in the $k$ th layer. Introducing next the interlayer interaction (8), the eigenvalues of the matrix (14) are obtained by applying a global Bethe 
ansatz consisting of the usual Bethe ansatz for each layer. The algebra is straightforward and one obtains

$$
Z_{M N K} \sim \max _{1 \leq n_{k} \leq N} \prod_{k=1}^{K}\left[\Lambda_{R}\left(n_{k}\right)+\Lambda_{L}\left(n_{k}\right)\right]^{M}
$$

with

$$
\begin{aligned}
& \Lambda_{R}\left(n_{k}\right)=e^{-2 h\left(n_{k+1}-n_{k-1}\right)} \omega_{1}^{N-n_{k}} \prod_{j=1}^{n_{k}}\left(\frac{\omega_{3} \omega_{4}-\omega_{5} \omega_{6}-\omega_{1} \omega_{3} z_{j}^{(k)}}{\omega_{4}-\omega_{1} z_{j}^{(k)}}\right) \\
& \Lambda_{L}\left(n_{k}\right)=e^{2 h\left(n_{k+1}-n_{k-1}\right)} \omega_{4}^{N-n_{k}} \prod_{j=1}^{n_{k}}\left(\frac{\omega_{1} \omega_{2}-\omega_{5} \omega_{6}-\omega_{2} \omega_{4} / z_{j}^{(k)}}{\omega_{1}-\omega_{4} / z_{j}^{(k)}}\right),
\end{aligned}
$$

where $\Lambda_{R}\left(\Lambda_{L}\right)$ refers to the eigenvalue for $\alpha_{m, 1, k}=+1(-1)$ 10 and, for each $1 \leq k \leq K$, the $n_{k}$ complex numbers $z_{j}^{(k)}, j=1,2, \ldots, n_{k}$ are the solutions of the Bethe ansatz equations

$$
e^{4 h\left(n_{k+1}-n_{k-1}\right)}\left(z_{j}^{(k)}\right)^{N}=(-1)^{n_{k}+1} \prod_{i=1}^{n_{k}}\left(\frac{B\left(z_{i}, z_{j}\right)}{B\left(z_{j}, z_{i}\right)}\right), \quad j=1,2, \cdots, n_{k}
$$

where $B\left(z, z^{\prime}\right)=\omega_{2} \omega_{4}+\omega_{1} \omega_{3} z z^{\prime}-\left(\omega_{1} \omega_{2}+\omega_{3} \omega_{4}-\omega_{5} \omega_{6}\right) z^{\prime}$. Note that the Bethe ansatz equation (17), which is obtained by imposing the cancellation of unwanted terms in the Bethe ansatz solution, differs from its usual form (see, for example, [14]) in the inclusion of the exponential factor involving $h$. Various special forms of this solution has been given previously $[7,9]$.

A dimer system with interlayer interactions: We now consider a $3 D$ lattice model consisting of layers of honeycomb dimer lattices. The dimers, which carry weights $u, v, w$ along the three honeycomb edge directions, are close packed within each layer and, in addition, interact between layers. For two dimers incident at the same $\{m, j\}$ site in adjacent layers, the interaction energy is given in Table I.

The $2 D$ honeycomb dimer system can be formulated as a five-vertex model, namely, an ice-rule model with the weights [14, 15]

$$
\left\{\omega_{1}, \omega_{2}, \omega_{3}, \omega_{4}, \omega_{5}, \omega_{6}\right\}=\{0, w, v, u, \sqrt{u v}, \sqrt{u v}\}
$$

The 5-vertex model is defined on a square lattice of size $M \times N$ mapping to an honeycomb lattice of $2 M N$ sites 14,15 . The mapping is such that the edge state $\alpha=+1(\beta=+1)$ 
corresponds to the presence of a $v(u)$ dimer. It can then be verified that the interlayer interaction given in Table I can be written precisely in the form of (8) [16], and therefore we can use the ice-rule model results.

Substituting (18) into (15), one obtains

$$
Z_{M N K} \sim u^{M N K} \max _{1 \leq n_{k} \leq N} \prod_{k=1}^{K} \prod_{j=1}^{n_{k}}\left(\frac{w}{u}+\frac{v}{u} z_{j}^{(k)}\right)^{M}
$$

with the Bethe ansatz solution

$$
z_{j}^{(k)}=e^{i \theta_{j}} e^{2 h\left(y_{k+1}-y_{k-1}\right)}, \quad j=1,2, \cdots, n_{k}
$$

where $e^{i \theta_{j}}$ are $n_{k}$ distinct $N$ th roots of $(-1)^{n_{k}+1}$. For a given $n_{k}$, the factor inside the parentheses in (19) attends its maximum if the $\theta_{j}$ 's lie on an arc crossing the positive real axis and extending from $-\pi\left(1-y_{k}\right) / 2$ to $\pi\left(1-y_{k}\right) / 2$. Using (3) this leads to the per-site free energy

$$
f_{K}=\ln u+\max _{-1 \leq y_{k} \leq 1} \frac{1}{K} \sum_{k=1}^{K} \frac{1}{2 \pi} \int_{-\pi\left(1-y_{k}\right) / 2}^{\pi\left(1-y_{k}\right) / 2} \ln \left(\frac{w}{u}+\frac{v}{u} e^{2 h\left(y_{k+1}-y_{k-1}\right)} e^{i \theta}\right) d \theta .
$$

This is our main result.

We have carried out analytic as well as numerical analyses of the free energy (21) for $K=3 \times$ integer. Here we summarize the findings. For $h=0$, the layers are decoupled and the property of the system is the same as that of the $2 \mathrm{D}$ system [14,15]. For large $h$, it is readily seen from Table I that the energetically preferred state is one in which each layer is occupied by one kind of dimers, $u, v$, or $w$, and the layers are ordered in the sequence of $\{w, v, u, w, v, u, \cdots\}$. It is also clear that for large $u, v$, or $w$, the system is also frozen with complete ordering of $u, v$, or $w$ dimers. These orderings are referred to as the $H, U, V$, and $W$ phases, respectively. The system can also be in two other phases. A $Y$ phase in which all layers have the same value of $y_{k}=y$ determined straightforwardly by maximizing (21), or

$$
w^{2}+v^{2}+2 w v \cos \left(\frac{\pi}{2}(1-y)\right)=u^{2}
$$


and an $I$ phase which is the $H$ phase with any of the $w, v$, or $u$ layers replaced by layers with $y_{k}=y$. If the $v$ layer is replaced by a $y$ layer so that the ordering is $\{w, y, u, w, y, u, \cdots\}$, for example, then $y$ is given by (22) with $v$ replaced by $v e^{4 h}$.

The phase diagram is found to be symmetric in $w, v$, and $u$. It is then convenient to plot the phase diagram using the coordinates

$$
X=\ln (v / w) \quad Y=(\sqrt{3})^{-1} \ln \left(v w / u^{2}\right)
$$

so that any interchange of the three variables $w, v$, and $u$ corresponds to a $120^{\circ}$ rotation in the $\{X, Y\}$ plane. The phase diagram for $h<h_{0}=0.2422995 \ldots$ is the same as in Fig. 2a but without the $H$ regime. Increasing the value of $h$ one finds the $H$ phase appear in $h_{0}<h<h_{1}=0.2552479 \ldots$ as shown in Fig. 2a. At $h=h_{1}$ the $I$ phase appears (Fig. $2 \mathrm{~b}$ ), with its region extending to infinity when $h$ reaches $h_{2}=(\ln 3) / 4=0.2746531 \ldots$ (Fig. 2c). When $h$ reaches $h_{3}=0.3816955 \ldots$ and higher, the $Y$ phase disappears completely as shown in Fig. 2d. All transitions are found to be of first-order except the transitions between the $\{U, V, W\}$ and $Y$ phases, and between the $I$ and $H$ phases, which are found to be of second-order with a square-root divergence in the specific heat.

In summary, we have presented the formulation of a general $3 D$ lattice model, and applied it to solve a realistic dimer system. The analysis can be extended to include dimer-dimer interactions within each layer [14], and further-neighbor interlayer interactions. Details of the present and further analyses will be presented elsewhere. Work has been supported in part by NSF Grant DMR-9313648 and by INTAS Grants 93-1324 and 93-0633. One of us (VP) thanks Prof. D. Kim for discussions. 


\section{REFERENCES}

[1] See R. J. Baxter, Exactly solved models in statistical mechanics (Academic, New York, 1982)

[2] M. Suzuki, Phys. Rev. Lett. 28, 507 (1972).

[3] A. B. Zamolodchikov, JETP 52, 325 (1980).

[4] R. J. Baxter, Commun. Math. Phys. 88, 185 (1983).

[5] V. V. Bazhanov and R. J. Baxter, J. Stat. Phys. 69, 453 (1992).

[6] F. Y. Wu and H. Y. Huang, Lett. Math. Phys. 29, 105 (1993).

[7] V. Popkov, Phys. Lett. A, 192, 337 (1994).

[8] A. E. Borovick, S. I. Kulinich, V. Popkov, Yu. M. Strzhemechny, Int. J. Mod. Phys. B 10, 443 (1996).

[9] V. Popkov and B. Nienhuis, J. Phys. A (to be published).

[10] See, for example, E. H. Lieb and F. Y. Wu, in Phase Transitions and Critical Phenomena Vol. I, Eds. C. Domb and M. S. Green (Academic Press, New York 1972).

[11] J. H. H. Perk and C. L. Schultz, Phys. Lett. A 84, 407 (1981).

[12] A. G. Izergin and V. E. Korepin, Commun. Math. Phys. 79, 303 (1981).

[13] See, for example, M. Wadati, T. Deguchi, and Y. Akutsu, Phys. Rep. 180, 247 (1989).

[14] H. Y. Huang, F. Y. Wu, H. Kunz, D. Kim, Physica A 228, 1 (1996).

[15] F. Y. Wu, Phys. Rev. 168, 539 (1968).

[16] To be more precise, (8) leads to Table I with $u u$ and $v v$ interactions $-\epsilon h$ instead of 0. But the two energies are equivalent, since the interacting $u u$ or $v v$ dimers are parallel covering the same A and B sites. 


\section{FIGURES}

FIG. 1. A three-dimensional lattice model consisting of layered vertex models.

FIG. 2. Phase diagrams of the dimer system. (a) $h_{0}<h<h_{1}$, (b) $h_{1}<h<h_{2}$, (c) $h_{2}<h<h_{3}$, (d) $h>h_{3}$. 


\section{TABLES}

TABLE I. Interaction energy between two dimers incident at the same $\{m, j\}$ site of adjacent layers. For example, a $v$ dimer in the $k$ th layer interacts with a $u$ dimer in the $(k+1)$ th layer with an energy $\epsilon h$. Here $\epsilon=+1(-1)$ if the site is in sublattice A (B).

\begin{tabular}{cccc}
\hline \hline layer $k \rightarrow k+1$ & $w$ & $v$ & $u$ \\
\hline$w$ & 0 & $-h$ & $h$ \\
$v$ & $h$ & 0 & $\epsilon h$ \\
$u$ & $-h$ & $\epsilon h$ & 0 \\
\hline \hline
\end{tabular}

